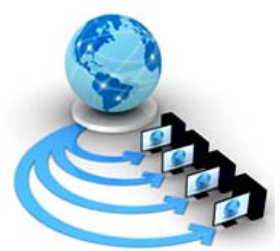

Volume 8, No. 7, July - August 2017

International Journal of Advanced Research in Computer Science

REVIEW ARTICLE

Available Online at www.ijarcs.info

\title{
PRE-PROCESSING THE BRAILLE IMAGE FOR IMPROVING OPTICAL BRAILLE RECOGNITION PERFORMANCE
}

\author{
Vishwanath Venkatesh Murthy \\ Department of Computer Science and Applications \\ Bangalore University \\ Bengaluru, India
}

\author{
M Hanumanthappa \\ Department of Computer Science and Applications \\ Bangalore University \\ Bengaluru, India
}

\begin{abstract}
Optical Braille Recognition (OBR), the automated software processes that are used in capturing and converting the Braille documents into text. The image data of embossed Braille plate captured using mobile camera or scanner is the input to OBR. Preprocessing is the first stage performed by OBR system. The Considerabletroubles in Image captured using camera, mobile or scanners include low-quality image due to irregular lightness during scanning, relatively low resolution of camera, impulse noise in the image, diverse gray-level values, introduce anelevated spatial frequency, angled or slanted image capturedas a resultof human error, deformation or warp of image document that continuedeprivation or disability of the dots, manifestation of uninvited dots, irregulargapin connecting dots and the cells representing character. These impulse noises are eliminated by applying various image enhancement techniques under preprocessing stage of OBR. The main mission of the paper is to study and provide a proportionallearning of diverse preprocessing techniques that are appliedto the Braille image by various researchers.
\end{abstract}

Keywords-Braille, Box filter, Gaussianfilter, Histogram Equalization, OBR, OCR, preprocessing, Smoothing, Spatialfilter.

\section{INTRODUCTION}

Braille is a language used by blind that consists of the pattern of combination of six raised dots called Braille cell that represent the text characters which are felt with the fingertips. The Braille cell might be stamped on single or double sided in the Braille manuscript.Maintaining such heavy Braille plates requires a lot of space and may get deteriorated if kept for long period. So there is a need to process the Braille document to text which can be stored easily for longer duration and can be distributed or reproduced on demand. The OBR (Optical Braille Recognition)is anapplicationthat performsthe conversionof Braille credentials to the human requisitetext. [3] The OBR structure has five distinct stages namely Image acquisition, Preprocessing, Image segmentation,Feature extraction as well as Conversion to Natural text.

This research paper is structured in 5 segments. Section-I introduce the Braille script withOBR systemalong with its stagesand detailed study on all preprocessing techniques has been discussed.In Section-II gives an insight of a Literature survey with the research work on these techniques. In SectionIII the challenges in image pre-processing of Braille document is discussed and the finallythe section-IV concludes the paper.

In image acquirementstage of the OBR,firstly Braille image is acquiredwith the devices likeMobile Phone camera, Digital camera or Scanners. The environment noise during image capture may produce low quality image due to unbalanced lightness, low resolution, diverse gray-level values, and elevated spatial frequency, and skewed or slanted image, deprivation of

the dots, unbalanced as well as uninvited dots and irregular space among dots. [10]The resultant imagenoise generally contains the Gaussian noise, impulse noise or salt and pepper noise.Enhancing and smoothing the acquired Braille information is performed in preprocessing stage of OBR which is a challenging task.Preprocessing progresses the probability of successful removal of noise introduced in image and recognition of degrades dots.

\section{- PREPROCESSING TECHNIQUES OF OBR}

To enhance the impulse noise in image, various Preprocessing Techniques can be applied namely Histogram Equalization, linear and Smoothing Spatial Filter, Box filter, Weighted Average filter, Gaussian filter, Median filter, Sharpening Spatial Filters, Unsharp Masking and Highboost Filtering, Laplacian filter, Roberts filter, Sobel Filter, Prewitt’s Filter.

The very first step in preprocessing is the image binarization. The acquired Braille image may be a color image is of no use as preprocessing techniques are applied over binary image. Hencethe color image is first required to be converted into binary image for further processing. Color image is a result of three grayscale images of $R, G$ and $B$ colors. In binary conversion, the RGB values for every individual pixel is taken and translated into a single value reflecting the brightness of that pixel as equation-1.

(Intensity of R + Intensity of G + Intensity of B)/3. - (1) 
The intensities are commonly are of range 30:60:10 for color combination of R:G:B components. The equation-2 is used to compute color image into gray scale.

$$
(0.3 \mathrm{R}+0.6 \mathrm{G}+0.1 \mathrm{~B}) / 3
$$

Histogram equalization process helps to enhance the intensity of pixels contrast in an image. The normal histogram $\mathrm{Pn}$ of a given image $\mathrm{v}$ is represented in equation-3.

$$
P n=\frac{\text { toal count of pixels with intensity } \mathrm{n}}{\text { total pixels in image }}
$$

Where, $\mathrm{n}=0,1 \ldots \mathrm{L}-1 . \mathrm{L}$ is intensity value 256 . The Transformation of the pixel intensities of histogram equalized image, $\mathrm{k}$ is represented by equation- 4 .

$$
T(k)=\text { floor }\left((\mathrm{L}-1) \sum_{n=0}^{k} \operatorname{Pn} \quad--(4)\right.
$$

Where, floor gives the rounded value of nearest integer.

Linear spatial filtering process helps to enhance image $\mathrm{v}$ by replacing the intensity value of every pixel with a value generated by a linear function applied on the values of nearby pixels. Different Linear Smoothing Filters used are Mean and Gaussian Filter, whereas Linear Edge enhancing filters used are i) Sobel Filter, ii) Prewitt Filter and iii) Laplace Filter.

For a point $\left(\mathrm{x}_{\mathrm{i}}, \mathrm{y}_{\mathrm{i}}\right)$, filter result $\mathrm{g}\left(\mathrm{x}_{\mathrm{i}}, \mathrm{y}_{\mathrm{i}}\right)$ is calculated by summing the coefficients of filter and encompasses pixels of an image using the equation-5.

$$
\begin{array}{r}
g\left(x_{i}, y_{i}\right)=w(-1,-1) f\left(x_{i}-1, y_{i}-1\right)+w(-1,0) f\left(x_{i}-1, y_{i}\right)+\ldots+ \\
w(0,0) f\left(x_{i}, y_{i}\right)+\ldots+w(1,1) f\left(x_{i}+1, y_{i}+1\right)
\end{array}
$$

Where, coefficient $\mathrm{w}(0,0)$, is the center coefficient of the filter. Linear spatial filtering for $\mathrm{m} \times \mathrm{n}$ size filter is specifiedin equation-6.

$$
g\left(x_{i}, y_{i}\right)=\sum_{s=-a}^{a} \sum_{t=-b}^{b} w(s, t) f\left(x_{i}+s, y_{i}+t\right) \quad \text {-- (6) }
$$

With no effect of blurring, image smoothing can be obtained by the equation-7.

$$
f\left(x_{i}, y_{i}\right)=\frac{1}{\mathrm{n}} \sum_{\mathrm{k}=1}^{\mathrm{n}} g_{n}\left(x_{i}, y_{i}\right) \quad--(7)
$$

Where, $g_{n}$ represents the pixel value and nrepresents the count of images of same scene. The correlation process moves the filter mask above the image covering each locality and finding the summation of products for these locations. The association of filter $\mathrm{w}\left(x_{i}, y_{i}\right)$ of size ' $m \times n$ ' is denoted by equation-8.

$$
\begin{gathered}
\mathrm{w}\left(x_{i}, y_{i}\right) \diamond f\left(x_{i}, y_{i}\right)= \\
\sum_{s=-a}^{a} \sum_{t=-b}^{b} w(s, t) f\left(x_{i}+s, y_{i}+t\right)
\end{gathered}
$$

Smoothing is the process of getting the average of the neighborhood pixels falling in the filter mask. The $\mathrm{m} \mathrm{x}$ weighted average filter is given by equation- 9 .

$$
g(x, y)=\frac{\sum_{s=-a}^{a} \sum_{t=-b}^{b} w(s, t) f(x+s, y+t)}{\sum_{s=-a}^{a} \sum_{t=-b}^{b} w(s, t)}
$$

The required parameters in the above formula are taken from the equation-9. An arithmetic mean enhances the corrupted image $\mathrm{g}(\mathrm{x}, \mathrm{y})$ of the area $\mathrm{S}_{\mathrm{xy}}$ by computing average value is given in the equation-10.

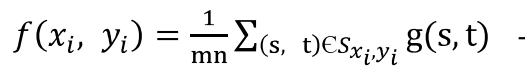

Mean filter reduces the noise by blurring the image. Whereas the median filter helps reducing the noise by less blurring effect comparing to mean filter is given by equation-11.

$$
f\left(x_{i}, y_{i}\right)=\underset{(s, t) \in S_{x_{i}, y_{i}}^{\text {median }}}{ }\{g(s, t)\}
$$

Gaussian filtering as in equation-12 helps remove noise by blurring image.

$$
\mathrm{G}(\mathrm{x})=\frac{1}{\sqrt{2 n \sigma^{2}}} e^{\left\{-\frac{x^{2}+y^{2}}{2 \sigma^{2}}\right\}}
$$

Fuzzy sets are also can be used for transforming the intensity. This process transforms the image into darker, gray or brighter as per the intensity of image. Transformed image v0 for an input $\mathrm{z} 0$ is given by equation- 13

$$
v_{0}=\frac{\mu_{\text {dark }}\left(z_{0}\right) \times v_{d}+\mu_{\text {gray }}\left(z_{0}\right) \times v_{g}+\mu_{\text {bright }}\left(z_{0}\right) \times v_{b}}{\mu_{\text {dark }}\left(z_{0}\right)+\mu_{\text {gray }}\left(z_{0}\right)+\mu_{\text {bright }}\left(z_{0}\right)}
$$

Fuzzy processing involves intensive calculations. Laplacian filter helps to sharpen the image. The symbol $\Delta$ is used to identify Laplacian filter $\mathrm{f}(\mathrm{x}, \mathrm{y})$.

The isotropic derivative operator Laplacian is the function of two variables defined by the equation-14. Laplacian function generally applied to an image under smoothing process with the approximation of a Gaussian smoothing filter for the purpose of reducing the noise. These derivatives are the linear operations. The equation-14 is implemented in the filter mask for smoothing process or correlation process.

$$
\begin{array}{r}
\Delta^{2} f=\frac{\partial^{2} f}{\partial x_{i},{ }^{2}}+\frac{\partial^{2} f}{\partial y_{i}{ }^{2}} \\
\frac{\partial^{2} f}{\partial x_{i}{ }^{2}}=f\left(x_{i}+1, y_{i}\right)+f\left(x_{i}-1, y_{i}\right)-2 f\left(x_{i}, y_{i}\right)- \\
\frac{\partial^{2} f}{\partial y_{i}{ }^{2}}=f\left(x_{i}, y_{i}+1\right)+f\left(x_{i}, y_{i}-1\right)-2 f\left(x_{i}, y_{i}\right)-
\end{array}
$$

The first variable in $\mathrm{x}$-coordinate direction is defined by equation-15, whereas second variable in $y$-direction is defined by equation-16. Finally the equation-17 gives the Laplacian equation for image sharpening.

$$
g\left(x_{i}, y_{i}\right)=f\left(x_{i}, y_{i}\right)+c\left[\Delta^{2} f\left(x_{i}, y_{i}\right)\right]--
$$

The edge detection algorithms normally use the various filtering techniques, amplification techniques and threshold operations.

\section{LITERATURE SURVEY}

Literature survey shows the preprocessing techniques used by various researchers. Preprocessing [1] can be grouped in major three arena as i) Translating the RGB image into corresponding grayscale ii) eliminating noise and iii) adjusting the image geometry. 
Jie Li [5] authorin the paper has used equation-2 to convert RGB image into grayscale image which evaluates the gray level charge ' $y$ ' forevery pixel [6].

For geometry adjustment [5], author Jie Li has physically drawn a $15 \times 10 \mathrm{~mm}$ cell rectangle. The algorithm identifies the same size cell rectangle from the scanned image. This identified cell region is compared with physically drawn box. This comparison is performed using equation-18 and 19 to estimate the rotation.

$$
\begin{aligned}
& x_{n}=\cos (\theta)\left(x_{p}-x_{0}\right)-\sin (\theta)\left(y_{p}-y_{0}\right)+x_{0}--(18) \\
& y_{n}=\sin (\theta)\left(x_{p}-x_{0}\right)+\cos (\theta)\left(y_{p}-y_{0}\right)+y_{0}--(19)
\end{aligned}
$$

Where, $\left(\mathrm{x}_{0}, \mathrm{y}_{0}\right)$ symbolize the co-ordinatevalues towardsthe center of rotation, $\left(x_{p}, y_{p}\right)$ and $\left(x_{n}, y_{n}\right)$ represents the newest and real pixel values in that order. The angle $\theta$ characterizethe estimated angle of rotation [5] [6].

Aisha Mousa has used the equation 09and 10 of average filtering routine for lessening the noise along withtechnique of contrast enrichment to amplify the strength oraltitude of recto dots [2].

L. Nian-feng authorhas usedanalgorithm of Hough transform [7], to estimate the tilted rotation angle that is spawned in image gaining process. The line data has been pre-marked and used inthe algorithm. The algorithm processes the supplied the array $A(r, \theta)$ and processes to get the minimum and maximum of $(r, \theta)$ using Hough transform which returns the required tilted angle value. The applied equation-20 represents the matrix of image rotation.

$$
\left[\begin{array}{l}
i^{\prime} \\
j^{\prime}
\end{array}\right]=\left[\begin{array}{c}
\cos \theta-\sin \theta \\
\sin \theta \cos \theta
\end{array}\right]\left[\begin{array}{l}
i \\
j
\end{array}\right] \quad--(20)
$$

The geometry rectification is done with adjusting rotation angle using the rotation matrix values of equation- 20 .

Mohd. Wajid [9] has performed the removal of the impulse or salt \& pepper noise using Median filtering method.

Lisa Wong [8] has made a simpler algorithm by introducing a pattern "half-characters" that represents the pattern of two columns of the target character. This Half character pattern was discoveredby implementing a pseudo code that is designed to handle the imagerow by row as a replacement over a regular 2 dimensional array.

Huaxun Zhang [4] has explained in paper of his work on geometry rectification.Author has used the linear regression method thatset rightthe tilt angle of the Braille document. Author has also performed the image enhancement using the histogram equalization method applied on each region.

\section{CHALlENGES IN PREPROCESSING THE BRAILLE DOCUMENT}

Very first challenge is of ensuring the physical geometry of the images and its consistentresolution. Most of the Author has applied the said preprocessing techniques on single sided Braille documents. Many documents are double-sided, printed inter-point. The recto and verso dot depressions of the Braille of one side can also appear interlaid on other side of the document.Applying the preprocessing techniques ontwo-sided Braille documents is a challenging task. Once the image preprocessing task gives the best results, rest of the OBR stages can be applied effectively. Hence in our next paper we will identify the best technique for preprocessing the Braille image.

\section{CONCLUSION}

Braille documents are converted to subsequent natural language character or text using Optical Braille recognition (OBR) system. In this paper we have concentrated our study only on preprocessing techniques. We have analyzed the different preprocessing methods used by various researchers. In our study we found that linear spatial filtering and Gaussian filtering are the best methods that give higher precision in preprocessing the Braille image. We also found that Majority of researchers hadput efforts on one-sided Braille paperto translate it intonatural text.We conclude that there is a lot of scope in improving the preprocessing stage of Braille. There is a lot of scope to work on conversion of double-sided Braille document which is a challenging task. In future work we plan to apply MATLAB on some best preprocessing techniques. We will concentrate our study to find out the best preprocessing method after evaluating the outcome results of MATLAB.

\section{REFERENCES}

[1] Abdul Malik S. Al-Salman, Ali El-Zaart, Yousef Al-Suhaibani, Khaled Al-Hokail, Abdu Gumaei, “Designing Braille Copier Based on Image Processing Techniques”, International Journal of Soft Computing and Engineering (IJSCE) ISSN: 22312307,Volume-4 Issue-5, November 2014

[2] Aisha Mousa, Hazem Hiary, Raja Alomari, and Loai Alnemer, "Smart Braille System Recognizer”, IJCSI International Journal of Computer Science Issues, Vol. 10, Issue 6, No 1, November 2013 ISSN (Print): 1694-0814, ISSN (Online): 1694-0784 www.IJCSI.org

[3] C. M. Ng, V. Ng and Y. Lau, "Regular feature extraction for recognition of Braille", Computational Intelligence and Multimedia Applications, 1999. ICCIMA '99. Proceedings. Third International Conference on, New Delhi, 1999, pp. 302306. doi: 10.1109/ICCIMA.1999.798547

[4] Huaxun Zhang, J. Li and J. Yin, "A Research on Paper-Mediated Braille Automatic Extraction Method," Intelligent Computation Technology and Automation (ICICTA), 2010 International Conference on, Changsha, China, 2010, pp. 328-331. doi: 10.1109/ICICTA.2010.145

[5] Jie Li and Xiaoguang Yan, "Optical Braille character recognition with Support-Vector Machine classifier," 2010 International Conference on Computer Application and System Modeling 
(ICCASM 2010), Taiyuan, 2010, pp. V12-219-V12-222, doi: 10.1109/ICCASM.2010.5622245

[6] Jie Li, Xiaoguang Yan and Dayong Zhang, "Optical Braille recognition with Haar wavelet features and Support-Vector Machine," 2010 International Conference on Computer, Mechatronics, Control and Electronic Engineering, Changchun, 2010, pp. 64-67. doi: 10.1109/CMCE.2010.5610062

[7] L. Nian-feng and W. Li-rong, "A kind of Braille paper automatic marking system," Mechatronic Science, Electric Engineering and Computer (MEC), 2011 International Conference on, Jilin, 2011, pp. 664-667. doi: 10.1109/MEC.2011.6025553

[8] L. Wong, W. Abdulla and S. Hussmann, "A software algorithm prototype for optical recognition of embossed Braille," Pattern
Recognition, 2004. ICPR 2004. Proceedings of the 17th International Conference on, 2004, pp. 586-589 Vol.2. doi: 10.1109/ICPR.2004.1334316

[9] Mohd. Wajid, M. Waris Abdullah and O. Farooq, "Imprinted Braille-character pattern recognition using image processing techniques," Image Information Processing (ICIIP), 2011 International Conference on, Himachal Pradesh, 2011, pp. 1-5. doi: 10.1109/ICIIP.2011.6108954

[10] T. Li, X. Zeng and S. Xu, "A Deep Learning Method for Braille Recognition", Computational Intelligence and Communication Networks (CICN), 2014 International Conference on, Bhopal, 2014, pp. 1092-1095. doi: 10.1109/CICN.2014.229. 